\title{
Analysis of Time and Frequency Domain Features of Accelerometer Measurements
}

\author{
Waltenegus Dargie \\ Chair for Computer Networks, Faculty of Computer Science \\ Technical University of Dresden 01062 Dresden, Germany \\ Email:waltenegus.dargie@tu-dresden.de
}

\begin{abstract}
This paper addresses the signal processing aspect of wireless sensor networks. It analyzes several time and frequency domain features of measurements that are taken from 3D accelerometer sensors. The measurements represent various types of movements related to humans and cars. The aim is to obtain quantitative as well as qualitative comparisons concerning the expression power of these features in the presence of various sources of uncertainties (calibration, placement of sensors, and time synchronization). For the qualitative analysis, we define fuzzy sets and fuzzy membership functions for all the features. Particular attention is given to the analysis of the existence of correlation between measurements of different sensor nodes. We will demonstrate that correlation coefficients of both time and frequency domain features exhibit high degrees of uncertainties. On the other hand, short time Fourier transformations (STFT) of all types of movements prove to be agnostic of various forms of measurement and calibration errors.
\end{abstract}

\section{INTRODUCTION}

A large number of applications in wireless sensor networks employ accelerometer sensors. Some of these applications use accelerometers to monitor the integrity of structures (bridges and building) [1], [2], [3], and [4]; transportation infrastructures [5] and [6]; supply-chain management [7], Healthcare [8], [9], [10] and [11], and earth quake and active volcano [12] and [13]. Almost all of these applications make use of modelbased digital signal processing to detect interesting events such as defects in bridges or abnormal drives, or the time and place at which objects breaks as a result of damage in wheels or major fractures in rails. Moreover, most of these applications analyze the existence of correlation between temporal and spatial features, for example, the existence of correlation between features representing movements or vibrations of suspender cables in bridges.

Unlike measurements taken from other types of sensors, the measurements from accelerometer sensors can be affected by three essential factors: calibration, placement, and orientation of the sensors. Two accelerometers sensors can deliver different reports about a movement of they differ in calibration, placement, or orientation or a combination of these. This is an essential observation in the context of wireless sensor network because of a basic assumption in spontaneous deployment.

In this paper, we provide analysis results of some measurements taken from 3-dimensional accelerometer sensors. The aim is to (1) give a comprehensive understanding of the time- and frequency-domain features that can be extracted from raw sensor data and, (2) examine their robustness despite calibration, placement, and orientation errors. While these features are exhaustively studied in the context of speech recognition and image processing, little is done in the context of wireless sensor networks, particularly with raw data that comes from wireless accelerometer sensors.

The remaining part of this paper is organized as follows: In Section III related work is summarized. In section III], the time and frequency domain features that are used for the analysis are introduced. In section IV, the methodology we used to acquire the sensor data is presented. In section $\mathrm{V}$, a detail account of the analysis as well as the results of the analysis are presented and discussed. Finally, in section $\mathrm{VI}$, concluding remarks and outlook to future work are given.

\section{RELATED WORK}

Several techniques and features have been employed in the past to process and classify data from accelerometer sensors. Huynh and Schiele [14] demonstrate how recognition rates can be improved by careful selection of features for different activities. Their experiment result suggests that the choice of a feature and a corresponding window length over which the feature is computed affect a recognition rate. Lukowicz et al. [15] investigate the existence of correlation in accelerometer signals to estimate various human activities. Likewise, Lee et al. [9] investigate the ambulatory condition (pulse) and movement (inclination of human body) using oximeter and triaxial accelerometer sensors.

Perhaps the most frequently employed technique in examining accelerometer signals is coherence. An interesting work related to this is the one carried out by Engin et al. [16] and [17] in which the presence of correlation between different axes of individual accelerometers and between different segments of the same limb (of a human body) was used to study the characteristics of tremor in patients with Parkinson's disease (PD). Data from triaxial accelerometers are collected from several PD patients and the existence of correlation (similar spectral features) is examined. The result suggests that tremor in PD is generated by multiple oscillatory circuits (in the central nervous system), which operate on similar frequencies. Coherence and spectral features are also 
used elsewhere to model and reason about various types of movements of objects.

Marin-Perianu et al. [18] experiment with an incremental correlation algorithm that enables wireless sensor nodes to determine whether they are traveling together (in supply chain management). The algorithm is implemented locally on a sensor node and the data processed is a real-time data series. The scalability of the algorithm is tested with respect to complexities related to communication, energy, memory and speed of execution.

The above approaches identify a set of time and frequency domain features and adopt a particular technique to recognize various activities and to examine the existence of correlation between these activities. Except Huynh and Schiele, who show how a recognition rate can be affected by the choice of features and their window length, the rest focus rather on the modeling aspect and employ a single technique (usually the coherence function) to recognize activities. These approaches, however, do not reveal much as to which techniques are more suitable to which activities and why. We build upon existing approaches, but address these and similar questions. Our approach enables application developers who intend to employ movement sensors, such as tilt and accelerometer sensors, to identify relevant features and correlation tools for recognizing interesting activities or events.

\section{Features}

The time- and frequency-domain features we consider are listed in table I. There are two reasons why we consider all these features: Firstly, feature extraction costs computational as well as communication resources. There is a relationship between the cost, the robustness and the expressive power of the features. Therefore, we closely examine the nature of these relationships. For example, all the time domain-features avoid the complexity of preprocessing - i.e., they do not require the laborious task of framing, windowing, filtering, Fourier transformation, liftering, and so on. Subsequently, they not only consume little processing power, but the algorithms can be directly deployed in resource constrained nodes. However, they are not robust to measurement and calibration errors. The second reason is our desire to support rapid prototyping by providing application developers the knowledge and experience concerning the type of features they can consider if they choose to employ accelerometer sensors. The features we analyze are listed in table [ ]

\section{Methodology}

The higher-level features that can be extracted from accelerometer sensors are subject to three sources of errors (uncertainties): calibration, non-optimal sensor placement, and packet los 1 . Time synchronization or the lack of it can be an additional source of uncertainty if one is interested in examining the existence of correlation between two sets of measurements. Apart from being dependent on the clock drift,

\footnotetext{
${ }^{1}$ This is without considering the imperfection in the sensors themselves.
}

\begin{tabular}{|l|l|l|}
\hline Domain & Feature & Remark \\
\hline \hline Time & Mean & \\
& Zero crossing rate & \\
& Maxima/Minima & \\
& Autocorrelation & \\
& Cross correlation & \\
& Linear correlation coefficient & \\
& Standard deviation & \multirow{2}{*}{ FFT and STFT } \\
\hline Frequency & Mean & \\
& Correlation & \\
& Spectral roll-off & \\
& Spectral centroid & \\
& Spectral flux & \\
\hline
\end{tabular}

TABLE I

TIME AND FREQUENCY DOMAIN FEATURES TO ANALYZE DATA FROM ACCELEROMETER SENSORS

\begin{tabular}{|l|l|}
\hline Processor & 32 bit ARM7 core \\
\hline Memory & 256K RAM/2M Flash \\
\hline Transceiver & $\begin{array}{l}\text { Chipcon CC2420 } \\
802.15 .4 \text { radio }\end{array}$ \\
\hline $\begin{array}{l}\text { Nominal power } \\
\text { consumption }\end{array}$ & $25-90 \mathrm{~mA}$ \\
\hline Size & $35 \times 25 \mathrm{~mm}$ \\
\hline
\end{tabular}

TABLE II

DESCRIPTION OF THE SUN SPOT SYSTEM

time synchronization is influenced by the transmission distance as well.

Our aim is to analyze how agnostic the time- and frequencydomain features are to these sources of errors. We consider different types of movements. We use two Sun Spot wireless sensor nodes containing 3-dimensional accelerometers (LIS3L02AQ) [19]. Table II] summarizes the specification of the nodes and table [II summarizes the features of the accelerometer sensors. The sensor nodes are simple, cheap and with limited resources. The maximum sampling frequency they support is $350 \mathrm{~Hz}$ (i.e., sampling every $\approx 3 \mathrm{~ms}$ ). The average communication delay between the sensor nodes and the remote sink is $4 \mathrm{~ms}$. To minimize packet loss, all data are logged to a remote computer at an average frequency of $150 \mathrm{~Hz}$. Sampling is carried out in a controlled environment to make sure that reading of all sensors takes place at the same time. Moreover, the active memory of each node is not fully overflown throughout the data acquisition period. The movement types we consider are summarized in section IV-A and IV-B

\section{A. Movement of people}

1) A single person: The sensors are attached to the thighs and wrists of a single person. The purpose is to investigate the the existence of a correlation between two

\begin{tabular}{|l|l|}
\hline Resolution & $0.5 \mathrm{mg}$ over $100 \mathrm{~Hz} \mathrm{BW}$ \\
\hline Range & $2 \mathrm{~g} / 6 \mathrm{~g}$ user selectable, full scale \\
\hline
\end{tabular}

TABLE III

FEATURE DESCRIPTION OF THE ACCELEROMETER SENSORS 
opposite direction 3D movements. The measurements are taken both from calibrated and uncalibrated sensors.

2) Two persons climbing up and down a staircase side by side (without intentional synchronization of steps): The sensors are attached to the thighs and wrists of two people climbing a staircase of 18 steps side by side. After the climb is over, the persons turn back and climb down the staircase. To consider the two opposite direction movements independently, the raw measurements are stored separately.

3) Two persons climbing up and down a staircase one after another (without intentional synchronization of steps): The sensors are attached to the test persons and the measurements are taken as in the previous case, but this time, one person is moving in front of the other.

4) Dancing: The sensors are attached to the right thighs of the test persons. They danced for about a minute. While the dance is a free and uncoordinated movement (with no premeditated pattern), there is, however, a body contact to mark the occurrence of certain distinct events.

5) Couch: The sensors are fixed on the bodies of two persons (once at the thighs and once at the waste). From a sitting position, these places represent the movements' center of gravity. The idea is to minimize the effect of undesirable activities (drinking a coffee from a mug; writing, etc.). The experiment measures the correlation of movements of two persons siting on a couch. The movements themselves are produced independently, but the movement of one person affects the movement of the other.

\section{B. Movement of Car}

1) Highway drive: a series of measurements are taken from the accelerometer sensors that are placed on the back and front seats of a car during a highway drive with an average speed of $120 \mathrm{Km} / \mathrm{H}$. The sensors themselves are not fixed to the seats, but are simply laying. Measurements from these sensors are taken to investigate the existence of correlation between the different parts of a car even though these parts react differently to accelerations and brakes as well as to the irregular surface underneath the car.

2) Highway drive: The sensors are attached to the seat belts of the driver and the front passenger, so that they can be closer to the center of gravity. However, exact alignment is not made. The reason for fixing the sensors this time is to measure the impact on the correlation due to the free movement of the sensors in the previous setting.

3) Free drive: In this setting the sensors are fixed in the front cabin of the car; they are calibrated and aligned to seats of the driver and the front passenger.

4) City drive: the sensors are placed inside the glove box at the front cabin of the car. Even though they are calibrated before hand, they are placed untethered. This is useful to measure the correlation between the measurements of these closely placed but unfixed nodes.
It is expected to observe strong correlation due to frequent accelerations and breaks as well as changes in the direction of drive.

\section{AnAlysis And Results}

\section{A. Time Domain Features}

1) Zero-crossing rate: Zero-crossing rate is a simple, straightforward and inexpensive feature to examine the similarity between two or more accelerometer sensors. It measures whether two sets of time series measurements exhibit similar patters. It is particularly useful to analyze measurements that are corrupted by noise. For example, a measurement with a high zero-crossing rate, i.e., the number of samples per second that cross the zero reference line, indicates that it is noisy. However, we observe that even for sensors having the same orientation, the calibration error results in a deviation of $\pm 40 / s$. Substituting the zero-crossing value by a mean valuecrossing, i.e., by defining a mean threshold that serves as a reference, results in a calibration error with a deviation between 2 and 15/s for individual axes and between 0 and 2/s for the absolute acceleration values of the individual measurements. Cross test of uncorrelated measurements, on the other hand, reveals that the overall calibration error is markedly bigger: between 7 and 50/s for the raw measurements and between 5 and $30 / \mathrm{s}$ for the absolute values.

2) Correlation coefficient: The correlation coefficient is another inexpensive but very useful tool to test whether two time-series readings exhibit similarity. Compared to the human movements, the movements related to the car drives yield bigger correlation coefficients, ranging from 0.4 to 0.9. Exception to this are the measurements taken from the sensors which lay fixed on the front and back seats of the car. In which case, it was not at all possible to establish correlation. Cross test of uncorrelated readings, on the other hand, results in correlation coefficients between 0.2 and \pm 0.1 only. The correlation coefficients related to human movements are markedly small, i.e., in the ranges of 0.2 and 0.3 . The correlation coefficients of the absolute values of all the measurements are notably better. The scenario that produces the highest correlation is the movements of people on the couch.

It must be noted, however, in the absence of time synchronization, the correlation coefficients of all movements are very small.

3) Cross correlation: The cross correlation is useful to measure the extent of the offset between two time-series measurements. This is particularly useful to model correlated movements that cannot be compared piecewise. A typical example is the correlation between the movements of people climbing up and down a staircase without synchronizing their steps. Intuitively, the movements should demonstrate strong correlations. However, due to the anatomy of the persons and the relative distance between the two people (back and forth), a sample-by-sample correlation is irrelevant. With the help of the cross correlation, we are able to detect and correct a mean offset value of \pm 1.4 s over all the three axes of the accelerometer sensors. 
4) Autocorrelation: Another approach to deal with measurements that cannot be compared piecewise is to test the linear correlation between two autocorrelation functions of two time series measurements. This feature performs very well to test uncorrelated movements instead of correlated movements.

Table IV summarizes the different time-domain features we consider. The features test both the presence and the absence of correlation between different measurements. In the second and the third column, the boundary signifies the width of the variance of similarity. We define fuzzy sets to qualitatively model the uncertainty associated with each feature. A trapezoid function is used to express membership. The beginning and end of the trapezoids are taken from the experiment results.

\begin{tabular}{|c|c|c|c|}
\hline Feature & $\begin{array}{l}\text { Deviation } \\
(\text { Correlated) }\end{array}$ & $\begin{array}{l}\text { Deviation } \\
\text { (Uncorrelated) }\end{array}$ & Uncertainty \\
\hline ZCR/MCR (s.T) & $0.0-15.0$ & $7-50$ & Medium \\
\hline ZCR/MCR (Abs.) & $0.0-2.0$ & $5.0-30.0$ & Low \\
\hline Mean value (s.T) & $\pm 0.5 g$ & $\pm 0.5 g$ & Unsuitable \\
\hline Mean value (Abs.) & $\pm 0.05 \mathrm{~g}$ & $\pm 0.5 g$ & Medium \\
\hline Corr. coeffi. (s.T. m) & $0.2-1$ & $<0.2$ & Medium/High \\
\hline Corr. coeffi. (s.T. c) & $0.4-1.0$ & $<0.4$ & Low \\
\hline \multicolumn{4}{|c|}{$\begin{array}{l}\text { Legend } \\
\text { s.T: Individual time series measurements }(\mathrm{x}, \mathrm{y}, \mathrm{z}) \\
\text { Abs.: The absolute values of the raw sensor measurements } \\
\text { m: Human movements } \\
\text { c: Car movements }\end{array}$} \\
\hline
\end{tabular}

TABLE IV

SUMMARY OF TIME DOMAIN FEATURES

\section{B. Frequency Domain Features}

All the frequency domain features require preprocessing and FFT. The resource consumption of these steps is discussed in [20] and [21]. Additional to these processes, we carry out frequency normalization (using a hamming window) to maximize the frequency resolution. This is because, unlike the time domain features, the frequency domain features heavily depend on domain knowledge, i.e., knowledge about the movements themselves.

1) Maxima: The $n$ - maxima of a frequency spectrum is used to compare the dominant frequencies of different measurements. To obtain a significant size of representative frequency samples, first the $i-t h$ maxima will be summed up and divided by the number of the total Maxima. Once this is done, comparison is made by selecting the $n-t h$ maximas and observing its deviation from the average maxima. For human movements, $n=100$ is sufficient, while for car $n$ is usually in the order of 1000 . This is because human movements contain low frequencies and the average distance for the first 100 maximas in frequency domain of the individual time series measurements lies between 0.7 and $3 \mathrm{~Hz}$. The distance of the maxima of the combination of the measurements from dissimilar movements lies between 1.6 and $4.9 \mathrm{~Hz}$. For car movement, the corresponding results are between 5 and 13 $\mathrm{Hz}$ for measurement with strong correlation and 20 and 30 for uncorrelated measurements.
2) Energy: The spectrum energy of a set of sensor readings reveals the spectrum's structure. In this context, the spectrum energy refers to the overall energy of the two readings being analyzed. To start with, the spectrum is divided into $n$ subbands and the portion of energy in each band is normalized by the overall energy of the spectrum. Correlation test is performed sub-band by sub-band comparison of different measurements. The stronger the existence of a correlation between the measurements, the lesser the difference between the sub-band energies. As a result the average difference of the sub-band energies is used to measure correlation. In our analysis, we find that the energy mass of similar movements of objects to be between 0.006 and 0.1 , while for uncorrelated, dissimilar movements the mass is between 0.2 and 0.9. Human movement is very difficult to categorize with the energy mass as its range is not strikingly different for similar and dissimilar movements.

3) Linear correlation coefficient: The frequency domain linear correlation coefficients reveal the presence of a strong correlation (between 0.6 and 0.99) for similar movements. Unfortunately, we observe (not infrequently), values above 0.6 even when the movements have nothing to do with each other. The best explanation for this is that all types of movements have high frequency components which reduce the significance of the bandpass frequencies, which are distinct from movement to movement. As a result, a large portion of the curves are similar and can wrongly be interpreted as being correlated. We attempt to reduce this effect by quantizing the measurements. We observe some improvement, testing the presence of similarity with linear correlation coefficients in the frequency domain remains feeble, nevertheless. Moreover, the quantization level is very much dependent on the measurements being compared or tested - the bigger the pick to pick amplitudes of the measurements, the larger should be the quantization level.

We investigate The change of frequencies over time as a measure of correlation. For this, we use the Short Time Fourier Transform (STFT), which is computed by dividing the sensor measurements into several overlapping frames. Each frame is then Fourier transformed, and the complex result is added to a matrix, recording the magnitude and phase of each point in time and frequency domain. As a scaling factor, we sum up the correlation coefficients and divide them by the size of the frequencies being considered. The test shows a marked difference between the movements of people and the movements of cars. For human movements, the results are on the average between 0.1 and 0.3 , which confirms the results we get in the time domain for the same data set. Cross tests of uncorrelated movements result in weighted linear correlation coefficients that range between 0.03 and 0.09. This much cannot be achieved in the time domain for the same data sets.

The result of car related movements are even better. While the correlation coefficients for similar movements are between 0.2 and 0.6 , for unrelated movements these are between 0.02 and 0.1 . This observation suggests that other frequency domain analysis such as coherence, can be significantly improved by 
considering STFT instead of the linear FFT.

4) Spectral roll-off: The spectral roll-off is another structural feature in the frequency domain in which only the Fourier transformation of the acceleration vectors are taken into account. In most cases, such as in speech recognition, it is usually customary to consider $\alpha=85 \%$. This, however, does not result in any significant difference between correlated and uncorrelated movements. Not unexpectedly, a significant porition of the energy of most movements is contained within the lower frequency components. Subsequently, we lower down the value of $\alpha$ to $60 \%$. As a result, similar movements reveal a roll-off distance that range from 0 to $4 \mathrm{~Hz}$ while the roll-off distance for uncorrelated movements are between 2.5 and $10 \mathrm{~Hz}$.

5) Spectral centroid: The spectral centroid is similar to the "first n-maxima" and indicates the relative location of the "centre of gravity" of the spectrum. It is computed as the weighted mean of the frequencies - the magnitudes of the frequencies being taken as weights. This scheme requires a precise knowledge of the movements being considered. The spectral centroid requires that the spectrum should be divided into several frames and the centroid of each frame is independently computed and piecewise comparison is performed on the centroids of the measurements being investigated.

Regardless of the movement types, division of the entire spectrum into five equal segments results in a centroid distance that ranges from 0.2 to $0.5 \mathrm{~Hz}$ for related movements and from 0.3 to $1.7 \mathrm{~Hz}$ for unrelated movements. By taking the absolute value of the spectrum, the related movements yield a better correlation, the centroid being tighter than the previous, i.e., from 0.2 to $0.3 \mathrm{~Hz}$.

6) Spectral Flux: The spectral flux is a measure of how quickly the power spectral changes. Ideally, similar movements should have a deviation of 0 flow. To compute the spectral flux of two measurements, one should compare measurements that have the same time duration. In the simplest case of considering the entire duration, we obtain a spectral flux that ranges from 0.0026 to 0.25 for similar movements, in which case 0.25 is rather a worst case. Otherwise, the spectral flux of similar movements is between 0 and 0.1 . On the contrary, the spectral flux of unrelated movements varies from 0.2 to 1 .

Table $\mathrm{V}$ summarizes our observation for the frequency domain analysis.

\section{CONCLUSION}

We considered various time and frequency domain features to analyze the existence of correlation between various measurements of accelerometer sensors. The measurements represent movements of humans and cars for various scenarios, both in calibrated and uncalibrated conditions. The time domain features we considered are: zero-crossing rate (corr., mean-value crossing rate), correlation coefficients, and cross correlations. The frequency domain features are: maxima and energy; correlation coefficients of both FFT and STFT, spectral roll-off, spectral centroids, and spectral flux. We find out that,

\begin{tabular}{|l|l|l|l|}
\hline Feature & $\begin{array}{l}\text { Deviation } \\
\text { (Correlated) }\end{array}$ & $\begin{array}{l}\text { Deviation } \\
\text { (Uncorrelated) }\end{array}$ & Uncertainty \\
\hline \hline Maxima (s.T. m) & $0.7-3 \mathrm{~Hz}$ & $1.4-3.9 \mathrm{~Hz}$ & High \\
\hline Maxima (Abs. m) & $0.7-3.5 \mathrm{~Hz}$ & $1.6-4.8 \mathrm{~Hz}$ & High \\
\hline Maxima (s.T. c) & $5-13 \mathrm{~Hz}$ & $20-30 \mathrm{~Hz}$ & Medium \\
\hline Maxima (Abs. c.) & $7-18 \mathrm{~Hz}$ & $11-25 \mathrm{~Hz}$ & Medium \\
\hline Energy (Abs. m.) & $0.0-0.02$ & $0.02-0.9$ & Medium \\
\hline Energy (Abs. c.) & $0.0-0.1$ & $0.2-0.9$ & Low \\
\hline Corr. (FFT) s.T. Abs. & $0.6-0.9$ & $0.6-0.9$ & Very high \\
\hline Corr. (STFT) Abs. m. & $0.1-0.3$ & $0.03-0.09$ & Low \\
\hline Corr. (STFT) Abs. c. & $0.2-0.6$ & $0.02-0.1$ & Low \\
\hline Spectral roll-off (Abs.) & $0-4 \mathrm{~Hz}$ & $2.5-10.0 \mathrm{~Hz}$ & Medium \\
\hline Spectral centroid (s.T) & $0.2-0.5 \mathrm{~Hz}$ & $0.3-1.7 \mathrm{~Hz}$ & Low \\
\hline Spectral Centroid (Abs.) & $0.2-0.3 \mathrm{~Hz}$ & $0.3-1.7 \mathrm{~Hz}$ & Low \\
\hline Spectral flux (Abs.) & $0.0-0.25$ & $0.2-0.1$ & Low \\
\hline $\begin{array}{l}\text { Legend } \\
\text { s.T: Individual time-series measurements (x, y, z) }\end{array}$ \\
$\begin{array}{l}\text { Abs.: The absolute values of the raw sensor measurements } \\
\text { m: Human movements } \\
\text { c: Car movements }\end{array}$
\end{tabular}

TABLE V

SUMMARY OF THE FREQUENCY DOMAIN FEATURES

the features extracted from the absolute values of the raw measurements are more robust to noise and calibration errors than the features extracted directly from the raw measurements. The frequency domain features that are least vulnerable to noise and exhibit the strongest expression power are the correlation coefficients of the absolute values of the short time Fourier transformations.

\section{REFERENCES}

[1] Krishna Chintalapudi, Tat Fu, Jeongyeup Paek, Nupur Kothari, Sumit Rangwala, John Caffrey, Ramesh Govindan, Erik Johnson, and Sami Masri. Monitoring civil structures with a wireless sensor network. IEEE Internet Computing, 10(2):26-34, 2006.

[2] Sukun Kim, Shamim Pakzad, David Culler, James Demmel, Gregory Fenves, Steven Glaser, and Martin Turon. Health monitoring of civil infrastructures using wireless sensor networks. In IPSN '07: Proceedings of the 6th international conference on Information processing in sensor networks, pages 254-263, New York, NY, USA, 2007. ACM.

[3] B. H. Koh and S. J. Dyke. Structural health monitoring for flexible bridge structures using correlation and sensitivity of modal data. Comput. Struct., 85(3-4):117-130, 2007.

[4] Ning Xu, Sumit Rangwala, Krishna Kant Chintalapudi, Deepak Ganesan, Alan Broad, Ramesh Govindan, and Deborah Estrin. A wireless sensor network for structural monitoring. In SenSys '04: Proceedings of the 2nd international conference on Embedded networked sensor systems, pages 13-24, New York, NY, USA, 2004. ACM.

[5] Prashanth Mohan, Venkata N. Padmanabhan, and Ramachandran Ramjee. Nericell: rich monitoring of road and traffic conditions using mobile smartphones. In SenSys '08: Proceedings of the 6th ACM conference on Embedded network sensor systems, pages 323-336, New York, NY, USA, 2008. ACM.

[6] Jakob Eriksson, Lewis Girod, Bret Hull, Ryan Newton, Samuel Madden, and Hari Balakrishnan. The pothole patrol: using a mobile sensor network for road surface monitoring. In MobiSys '08: Proceeding of the 6th international conference on Mobile systems, applications, and services, pages 29-39, New York, NY, USA, 2008. ACM.

[7] Mateusz Malinowski, Matthew Moskwa, Mark Feldmeier, Mathew Laibowitz, and Joseph A. Paradiso. Cargonet: a low-cost micropower sensor node exploiting quasi-passive wakeup for adaptive asychronous monitoring of exceptional events. In SenSys '07: Proceedings of the 5th international conference on Embedded networked sensor systems, pages 145-159, New York, NY, USA, 2007. ACM. 
[8] Stinne Aalökke Ballegaard, Thomas Riisgaard Hansen, and Morten Kyng. Healthcare in everyday life: designing healthcare services for daily life. In CHI '08: Proceeding of the twenty-sixth annual SIGCHI conference on Human factors in computing systems, pages 1807-1816, New York, NY, USA, 2008. ACM.

[9] Young-Dong Lee, Sang-Joong Jung, Yong-Su Seo, and Wan-Young Chung. Measurement of motion activity during ambulatory using pulse oximeter and triaxial accelerometer. In ICCIT '08: Proceedings of the 2008 Third International Conference on Convergence and Hybrid Information Technology, pages 436-441, Washington, DC, USA, 2008. IEEE Computer Society.

[10] Sung Won Yoon, Se Dong Min, Yong Hyeon Yun, Seungpyo Lee, and Myoungho Lee. Adaptive motion artifacts reduction using 3-axis accelerometer in e-textile ecg measurement system. J. Med. Syst., 32(2):101-106, 2008

[11] Josip Music, Roman Kamnik, Vlasta Zanchi, and Marko Munih. Human body model based inertial measurement of sit-to-stand motion kinematics. WTOS, 7(3):156-164, 2008

[12] Geoffrey Werner-Allen, Konrad Lorincz, Matt Welsh, Omar Marcillo, Jeff Johnson, Mario Ruiz, and Jonathan Lees. Deploying a wireless sensor network on an active volcano. IEEE Internet Computing, 10(2):18-25, 2006.

[13] Geoff Werner-Allen, Konrad Lorincz, Jeff Johnson, Jonathan Lees, and Matt Welsh. Fidelity and yield in a volcano monitoring sensor network. In OSDI '06: Proceedings of the 7th symposium on Operating systems design and implementation, pages 381-396, Berkeley, CA, USA, 2006. USENIX Association.
[14] Tâm Huynh and Bernt Schiele. Analyzing features for activity recognition. In sOc-EUSAI '05: Proceedings of the 2005 joint conference on Smart objects and ambient intelligence, pages 159-163, New York, NY, USA, 2005. ACM.

[15] Jamie A. Ward, Paul Lukowicz, Gerhard Troster, and Thad E. Starner. Activity recognition of assembly tasks using body-worn microphones and accelerometers. IEEE Trans. Pattern Anal. Mach. Intell., 28(10):1553-1567, 2006.

[16] Mehmet Engin, Serdar Demirağ, Erkan Zeki Engin, Gürbüz Çelebi, Fisun Ersan, Erden Asena, and Zafer Çolakoğlu. The classification of human tremor signals using artificial neural network. Expert Syst. Appl., 33(3):754-761, 2007.

[17] Noeël L.W. Keijsers, Martin W.I.M. Horstink, and Stan C.A.M. Gielen. Ambulatory motor assessment in parkinsons disease. Movement Disorder Society, 21(1):34-44, 2006.

[18] Raluca Marin-Perianu, Johann Hurink, and Pieter Hartel. A generalized clustering algorithm for dynamic wireless sensor networks. In ISPA '08: Proceedings of the 2008 IEEE International Symposium on Parallel and Distributed Processing with Applications, pages 863-870, Washington, DC, USA, 2008. IEEE Computer Society.

[19] Sun Microsystem: http://www.sunspotworld.com/. Project Sunspot.

[20] Waltenegus Dargie. Adaptive audio-based context recognition. IEEE Transaction on systems, man, and cybernetics-Part A: Systems and Humans.

[21] Waltenegus Dargie and Tobias Tersch. Recognition of complex settings by aggregating atomic scenes. IEEE Intelligent Systems, 23(5):58-65, 2008. 